BULLETIN OF THE

AMERICAN MATHEMATICAL SOCIETY

Volume 77, Number 1, January 1971

\title{
CURVATURE AND DIFFERENTIABLE STRUCTURE ON SPHERES 1
}

\author{
BY ERNST A. RUH
}

\section{Communicated by S, Sternberg, July 2, 1970}

1. Introduction. The purpose of this note is to outline a proof of the following result: A simply connected, complete, riemannian manifold whose curvature tensor $R$ is sufficiently close to the curvature tensor $R_{0}$ of the standard sphere $S$ of the same dimension is diffeomorphic to $S$. Traditionally, the proximity of $R$ and $R_{0}$ has been measured in terms of the sectional curvature as follows: A riemannian manifold is called $\delta$-pinched if the sectional curvature $K$ satisfies the condition $\delta<K \leqq 1$. Using this concept, Gromoll [4] and Calabi proved the following diffeomorphism theorem: There exists a sequence $\delta_{n}$ with $\lim \delta_{n}=1$ as $n$ increases such that a $\delta_{n}$-pinched simply connected riemannian manifold $M$ of dimension $n$ is diffeomorphic to the sphere $S^{n}$.

In order to express the main condition of the diffeomorphism theorem independently of dimension, we introduce a different measurement for the proximity of the curvature tensors $R$ and $R_{0}$ of the manifolds $M$ and $S^{n}$ respectively. To formulate this condition we think of the riemannian curvature tensor as a selfadjoint, linear map $R: V \wedge V \rightarrow V \wedge V$, where $V \wedge V$ denotes the exterior product of the tangent space with itself. A riemannian manifold is called strongly $\delta$-pinched, if the eigenvalues $\lambda$ of the above linear map at every point of $M$ satisfy the condition $\delta<\lambda \leqq 1$.

2. Statement of result. In previous studies the pinching constant depended on the dimension of the manifold. However, the introduction of strong $\delta$-pinching has the following advantage: The constant $\delta$ in the theorem below is independent of the dimension of the manifold.

TheOREM. There exists a constant $\delta \neq 1$ such that a complete, simply connected, strongly $\delta$-pinched riemannian manifold is diffeomorphic to the standard sphere of the same dimension.

AMS 1969 subject classifications. Primary 5372.

Key words and phrases. Alexandrov-Rauch-Toponogoff comparison theorem, \&pinching, Gauss map, Morse theory, strong $\delta$-pinching.

1 Research supported by NSF Grant No. GP 20628. 
The main idea of the following proof is new. However, methods similar to those employed by Rauch [7], Berger [1], [2], Klingenberg [5], [6], Gromoll [4], and Cheeger [3] have been adapted to obtain the necessary estimates.

3. Outline of proof. We can suggest an idea of the proof by observing the Gauss map $g: M \rightarrow S^{n}$ that exists in case $M$ is an $n$-dimensional manifold embedded in euclidian space $E^{n+1}$. Of course, the map $g$ sending $x \in M$ into the unit normal vector at $x$ translated to a fixed point $x_{0}$ is well defined because parallel translation in $E=M \times E^{n+1}=\tau(M) \oplus \nu(M)$, where $\tau(M)$ and $\nu(M)$ denote tangent and normal bundle respectively, is independent of the path. In addition, $g$ is a local diffeomorphism as long as the derivative $D_{X} n$ of the unit normal vector field $n$ in any direction $X \neq 0$ is nonzero.

In the general case the normal bundle is not available; however, we replace it by a trivial line bundle $\epsilon$ and define a flat connection $\nabla^{\prime}$ on $E=\tau(M) \oplus \epsilon$. At this point a map $f: M \rightarrow S^{n}$ is defined by replacing the normal vector field by a section $e$ of length one in $\epsilon$; i.e., the image $f(x)$ is obtained by parallel translation of $e(x)$ to the fibre $E^{n+1}$ over a fixed point $x_{0}$. Again, $f$ is a local, and since $M$ is simply connected, a global diffeomorphism as long as $\nabla_{X}^{\prime} e \neq 0$. Therefore, the proof consists of defining a flat connection $\nabla^{\prime}$ on $\tau(M) \oplus \epsilon$ and checking $\nabla_{x}^{\prime} e \neq 0$.

The first step in the construction of $\nabla^{\prime}$ is to define a connection $\nabla^{\prime \prime}$ in $E$ with small curvature as follows:

$$
\nabla_{X}^{\prime \prime} e_{i}=\nabla_{X} e_{i}-\frac{1}{2}(1+\delta)\left\langle X, e_{i}\right\rangle e, \quad \nabla_{X}^{\prime \prime} e=\frac{1}{2}(1+\delta) X,
$$

where $\nabla$ denotes the riemannian connection in the tangent bundle $\tau(M) ; e_{i}, i=1,2, \cdots, n$, denotes a moving orthonormal frame in $\tau(M)$; and $e$ is a section of length one in $\epsilon$. The curvature of $\nabla^{\prime \prime}$ can be estimated in terms of $\delta$. The idea for the definition of $\nabla^{\prime \prime}$ originates from the following observation: In case $M$ is the standard sphere embedded in $E^{n+1}$, the covariant derivative defined above is nothing but the ordinary derivative in $E^{n+1}$.

In the next step, $\nabla^{\prime \prime}$ is used to construct a cross section $u^{\prime}$ in the principal bundle of $n+1$-frames associated to $E$. The results necessary for this construction are compiled in the first four chapters of [4]. The proofs are based on the Alexandrov-Rauch-Toponogoff comparison theorem and the Morse critical point theory. In particular, we use the representation of $M$ as the union $M_{0} \cup M_{1}$ of two balls representing upper and lower hemisphere. On $M_{0}$ we define a cross section $u_{0}$ by moving a fixed $n+1$-frame $u_{0}\left(q_{0}\right)$ chosen over the center $q_{0}$ of $M_{0}$ by parallel translation with respect to $\nabla^{\prime \prime}$ along geodesic 
rays to points in $M_{0}$. On $M_{1}$ we define first $u_{1}\left(q_{1}\right)$ by parallel translation of $u_{0}\left(q_{0}\right)$ along a shortest geodesic to $q_{1}$, the center of $M_{1}$. Subsequently, $u_{1}$ is defined on $M_{1}$ by translation along geodesic rays. On $C=M_{0} \cap M_{1}$ the sections $u_{0}$ and $u_{1}$ may not coincide, but the distance in the fibre can be estimated in terms of the pinching constant $\delta$. Therefore, for $\delta$ close enough to 1 , the sections $u_{0}$ and $u_{1}$ can be modified to yield a differentiable cross section $u^{\prime}$ on $M$. At this point, let $\nabla^{\prime}$ denote the flat covariant derivative in $E=\tau(M) \oplus \epsilon$ corresponding to $u^{\prime}$.

It remains to be shown that $\nabla_{X}^{\prime} e \neq 0$. The result follows because for $\delta$ close to 1 , the difference of $\nabla^{\prime}$ and $\nabla^{\prime \prime}$ is small and

$$
\left\|\nabla_{X}^{\prime \prime} e\right\|=\frac{1}{2}(1+\delta)\|X\| \sim\|X\| \text {. }
$$

The details, as well as an estimate for the pinching constant $\delta$, will be furnished in a subsequent paper.

\section{BIBLIOGRAPHY}

1. M. Berger, Les variêtés riemanniennes dont la courbure satisfait certaines conditions, Proc. Internat. Congress Math. (Stockholm, 1962). Inst. Mittag-Leffler, Djursholm, 1963, pp. 447-456. MR 31 \#695.

2. - Les varietés Riemanniennes (1/4)-pincees, Ann. Scuola Norm. Sup. Pisa (3) 14 (1960), 161-170. MR 25 \#3478.

3. J. Cheeger, Pinching theorems for a certain class of Riemannian manifolds, Amer. J. Math. 91 (1969), 807-834.

4. D. Gromoll, Differenzierbare Strukturen und Metriken positiver Krilmmung auf Sphären, Math. Ann. 164 (1966), 353-371. MR 33 \#4940.

5. W. Klingenberg, Contributions to Riemannian geometry in the large, Ann. of Math. (2) 69 (1959), 654-666. MR 21 \#4445.

6. - Über Riemannsche Mannigfaltigkeiten mit positiver Kriummung, Comment. Math. Helv. 35 (1961), 47-54. MR 25 \#2559.

7. H. E. Rauch, A contribution to differential geometry in the large, Ann. of Math. (2) 54 (1951), 38-55. MR 13, 159.

Purdue University, Lafayette, Indiana 47907 